\title{
Tri-modal In vivo Imaging of Pancreatic Islets Transplanted Subcutaneously in Mice
}

\author{
Sayuan Liang, ${ }^{1,2,3}$ Karim Louchami, ${ }^{1,4}$ Bryan Holvoet, ${ }^{5}$ Rein Verbeke, ${ }^{6}$ \\ Christophe M. Deroose, ${ }^{5}$ Bella Manshian, ${ }^{1}$ Stefaan J. Soenen, ${ }^{1}$ Ine Lentacker, ${ }^{6}$ \\ Uwe Himmelreich ${ }^{1}$ \\ ${ }^{1}$ Biomedical MRI, Department of Imaging \& Pathology, University of Leuven, Leuven, Belgium \\ ${ }^{2}$ Bio-Imaging Lab, Faculty of Pharmaceutical, Biomedical and Veterinary Sciences, University of Antwerp, Antwerp, Belgium \\ ${ }^{3}$ Philips Research China, Shanghai, China \\ ${ }^{4}$ Laboratory of Experimental Hormonology, Université Libre de Bruxelles, Bruxelles, Belgium \\ ${ }^{5}$ Nuclear Medicine \& Molecular Imaging, Department of Imaging \& Pathology, University of Leuven, Leuven, Belgium \\ ${ }^{6}$ Laboratory for General Biochemistry and Physical Pharmacy, Ghent University, Ghent, Belgium
}

\begin{abstract}
Purpose: Transplantation of pancreatic islets (PIs) is a promising therapeutic approach for type 1 diabetes. The main obstacle for this strategy is that the outcome of islet engraftment depends on the engraftment site. It was our aim to develop a strategy for using non-invasive imaging techniques to assess the location and fate of transplanted PIs longitudinally in vivo.

Procedures: In order to overcome the limitations of individual imaging techniques and crossvalidate findings by different modalities, we have combined fluorine magnetic resonance imaging (F-19 MRI), fluorescence imaging (FLI), and bioluminescent imaging (BLI) for studying subcutaneously transplanted Pls and beta cell-like cells (INS-1E cell line) in vivo. We optimized the transduction (using lentiviral vectors) and labeling procedures (using perfluoro crown ether nanoparticles with a fluorescence dye) for PIs and INS-1E cell imaging.

Results: The feasibility of using the proposed imaging methods for PI assessment was demonstrated both in vitro and in vivo. Our data suggested that F-19 MRI is suitable for highresolution localization of transplanted cells and PIs; FLI is essential for confirmation of contrast localization by histology; and BLI is a reliable method to assess cell viability and survival after transplantation. No significant side effects on cell viability and function have been observed. Conclusions: The proposed tri-modal imaging platform is a valuable approach for the assessment of engrafted PIs in vivo. It is potentially suitable for comparing different transplantation sites and evaluating novel strategies for improving PI transplantation technique in the future.
\end{abstract}

Key words: Pancreatic islet, Transplantation, Subcutaneous, Multimodal imaging, Fluorine-19 magnetic resonance imaging, Fluorescent imaging, Bioluminescent imaging

Electronic supplementary material The online version of this article (https:// doi.org/10.1007/s11307-018-1192-0) contains supplementary material, which is available to authorized users.

Correspondence to: Uwe Himmelreich; e-mail uwe.himmelreich@med.kuleuven.be

\section{Introduction}

Patients with type 1 diabetes (T1D) suffer from hyperglycemia due to the loss of insulin-producing beta cells in the pancreatic islets (PIs) caused by an autoimmune attack 
mediated by $\mathrm{T}$ cells [1]. Current clinical routine therapy, such as insulin injections or insulin pumps, can control blood glucose levels to some extent. However, insulin administration is often not sufficient for T1D patients. Most of those patients will still have abnormally high levels of blood glucose and are at risk for long-term complications like blindness, neuropathy, kidney disease, and others [2]. As a possible alternative therapeutic approach, engraftment of pancreatic islets is considered, which is currently assessed in selected patient populations.

After the first introduction of PI and beta cell transplantation in rats [3] and further successful clinical trials on T1D patients [4], many studies and technical improvements have been implemented. This includes the process of PI isolation from donors, strategies for immunosuppression, and management of immunomodulation of recipients as well as antiinflammatory drugs after surgery, both in a pre-clinical and clinical setting $[5,6]$. Some of these initial results have already been tested in clinical trials or are in the process of becoming real treatment options for patients $[7,8]$.

One of the main remaining obstacles for PI transplantation is the variable outcome of islet engraftment, with a strong influence on the site of transplantation. Currently, the liver serves as the most practical clinical transplantation site for PIs or beta cells [9] with several completed clinical trials using intraportal transplantation [10, 11]. Other potential sites for PI or beta cell transplantation have been proposed, such as subcutaneous tissue, the kidney (subcapsular), the omentum, the muscle, and the anterior chamber of the eye $[12,13]$. All of these transplantation sites have their own strengths and limitations in terms of surgery, impact on cell metabolism, tissue perfusion, or immunological aspects [14]. One of the key steps to validate islet engraftment is longitudinal, in vivo monitoring of the exact location and fate of the transplanted PIs, with the possibility of quantification without invasive intervention.

Non-invasive imaging techniques are increasingly available for longitudinal in vivo tracking of transplanted islets [15]. Different imaging modalities have been proposed for the visualization of endogenous and exogenous PIs. Bioluminescence imaging (BLI) requires prior engineering of islets to express a luciferase gene to provide a quantitative measure for viable islets $[16,17]$. Fluorescent imaging (FLI) requires either cells that express fluorescent reporter genes or cells preloaded with fluorescent dyes and was successfully used for continuous monitoring of surviving PIs [12]. Radionuclide imaging has also been used for PI and beta cell imaging after pre-labeling with radiotracers $[18,19]$ or by using transfected/ transduced cells for reporter gene-based approaches [20]. However, PET and SPECT have a relatively low resolution and require radioactive tracers. For magnetic resonance imaging (MRI), different contrast agents have been used for pre-labeling and further enhancement of MR contrast for in vivo islet and beta cell tracking. This includes superparamagnetic iron oxide (SPIOs) nanoparticles and paramagnetic gadolinium chelates, resulting in retrievable signal for up to 24 weeks [21-23] and 65 days [24], respectively. Recently, cell tracking using fluorine containing contrast agents and F-19 MR imaging has opened new perspectives for quantitative in vivo islet imaging $[25,26]$. Advantages and disadvantages of the different imaging techniques can be linked to the transplantation site. For example, optical imaging (BLI or FLI) is limited to superficial tissues due to its limited signal depth penetration and light scattering. But, it can provide information on in vivo cell viability (BLI). F-19 MRI is superior in terms of specificity and potential quantification of transplanted islets or cells due to the lack of MRdetectable F-19 signal in biological tissues. Its downside is its relatively low sensitivity so that several thousand cells are required for sufficient MR signal intensity [27].

To overcome the limitations in sensitivity, resolution, and specificity of individual imaging techniques, multimodal imaging serves as a potential solution for cross-validation, better visualization, and follow-up of the fate of transplanted islets and beta cells in vivo. In this study, we aimed to combine BLI, FLI, and F-19 MRI techniques in a multimodal approach for in vivo monitoring of transplanted rodent islets and beta cell-like cell lines. Hereby, we have either used transduced cell lines or cells pre-labeled with F19 contrast agents.

\section{Materials and Methods}

\section{Fluorinated Particles and Lentiviral Vector}

For pre-labeling purpose, cationic perfluoro-15-crown-5 ether (PFCE) particles (particle concentration: $4.5 \times 10^{9}$ particles/ ml, particle mean size: $0.5 \pm 0.1 \mu \mathrm{m})$ were prepared as previously reported (see electronic supplementary material (ESM)) [28].

A lentiviral vector (LV) carrying the human elongation factor $1 \alpha(\mathrm{hEFl} \alpha)$ promoter to drive triple flag tagged firefly luciferase (3flagFluc) and a puromycin resistance gene (PuroR) was used for islets/cells transduction as described in [29].

\section{Rodent Pancreatic Islets and Cell Lines}

The PIs used in this study were isolated from Wistar rats (female, 8-10 weeks, 200-250 g) using the collagenase digestion method (see ESM) [30].

The pancreatic beta cell-like INS-1E cell line, used at passage 52, was a kind gift from C.B. Wollheim (Department of Cell Physiology and Metabolism, University Medical Center, Geneva, Switzerland). For the preparation protocol, see ESM.

\section{Lentiviral Vector Transduction Experiments}

In order to generate firefly luciferase expressing INS-1E cells, untreated cells $\left(\sim 4 \times 10^{5}\right.$ cells/well $)$ were seeded into six-well plates (Sigma) ESM material). 


\section{Cell Labeling Using PFCE Particles}

INS-1E cell labeling experiments were performed using different final fluorine concentrations $(10,15,20,30$, and $50 \mathrm{mM})$ with different incubation times (4 and $24 \mathrm{~h}$ ) (see ESM).

For optimization of PI labeling, different incubation times $(4,8,10,20,24$, and $48 \mathrm{~h})$ were used in the presence of PFCE particles, resulting in a $20 \mathrm{mM}$ fluorine concentration. Similar to the INS-1E cells, PIs were handpicked and divided for different in vitro assays such as fluorescence microscopy, confocal microscopy, and NMR spectroscopy after incubation and washing. Unlabeled PIs/cells were always included as negative controls in all experiments.

After optimization of PFCE labeling protocols, studies on the release of the fluorine labels from pre-labeled PIs and INS-1 cells were also conducted (see ESM).

\section{F-19 NMR Experiments}

All F-19 NMR experiments were performed using a 400MHz Bruker Avance II NMR spectrometer (Bruker Biospin, Rheinstetten, Germany) equipped with a $5-\mathrm{mm}$ broadband probe, which was also tunable to $376.5 \mathrm{MHz}$ for F-19 NMR spectroscopy (see ESM).

\section{Confocal Microscopy}

Confocal microscopy images (including z-stacked images) were acquired using a Nikon A1R Eclipse confocal microscope (Nikon, Brussels, Belgium) to verify the internalization of PFCE by cells of the PIs (see ESM).

\section{In vitro Islet Viability and Functionality Assessment}

To confirm the viability and function of PIs after labeling and transduction, trypan blue staining, insulin content measurement, and glucose-stimulated insulin secretion tests were performed (see ESM).

\section{In vitro BLI, FLI, and F-19 MRI}

In vitro BLI, FLI, and F-19 MRI were carried out to determine the feasibility of imaging pre-labeled and transduced PIs/cells with multimodal imaging and to determine the advantages and disadvantages of each technique. For BLI and FLI, phantoms were prepared by placing PIs $(n \sim$ $50)$ or INS-1E cells $\left(n \sim 3 \times 10^{5}\right)$ in six-well plates containing PBS. All BLI and FLI experiments were acquired using an IVIS Spectrum optical imaging system (PerkinElmer, Massachusetts, USA). For BLI, D-luciferin, dissolved in PBS $(15 \mathrm{mg} / \mathrm{ml})$, was also added prior to the imaging sessions. Consecutive frames were acquired (one per min) until the maximum signal intensity was reached. For FLI acquisition, the EPI mode was used with an excitation wavelength of $648 \mathrm{~nm}$ and an emission wavelength of $670 \mathrm{~nm}$ for the DiD fluorescence dye according to the default setting of the system's software (Living Imaging, Perkin Elmer).

For F-19 MRI, phantoms were prepared using $2 \%$ agarose (Sigma) suspensions in 0.5-ml Eppendorf tubes (Eppendorf, Rotselaar, Belgium). Hereby, Eppendorf tubes were either filled with PFCE-labeled PIs $(n \sim 100)$, PFCElabeled INS-1E cells $\left(n \sim 5 \times 10^{5}\right)$, pure PFCE particles (final F-19 concentration: $25 \mathrm{mM}$, positive control), or PBS (negative control). The Eppendorf tubes were then placed parallel in a $25-\mathrm{ml}$ plastic cylinder with a $2.8-\mathrm{cm}$ diameter, which also contained $2 \%$ agarose.

All MR experiments were performed using an 9.4-T Bruker Biospec small animal MR scanner (Bruker Biospin, Ettingen, Germany) equipped with a home-built saddle-shaped surface coil, which was tuneable and matchable to both the F-19 and H-1 resonances [26]. Both H-1 and F-19 MR images were acquired using a 2D RARE sequence with the following acquisition parameters: TE $=15.9 \mathrm{~ms}, \mathrm{TR}=3 \mathrm{~s}(\mathrm{H}-1) / 1 \mathrm{~s}(\mathrm{~F}-19), \mathrm{RARE}$ factor $=8, \mathrm{FOV}=6.4 \times 6.4 \mathrm{~cm}$, matrix size $=256 \times 256(\mathrm{H}-1) /$ $64 \times 64(\mathrm{~F}-19), \mathrm{NA}=2(\mathrm{H}-1) / 256(\mathrm{~F}-19)$, slice thickness $=$ $0.5 \mathrm{~mm}(\mathrm{H}-1) / 2.5 \mathrm{~mm}$ (F-19), total acquisition time $\sim 40 \mathrm{~min}$.

\section{Animal Model}

All animal experiments were performed in accordance with national and European regulations and approved by the local Animal Ethics Committee of KU Leuven. In total, 11 Swiss Nude mice (female, 7-9 weeks old, Charles River, Chatillon-sur-Chalaronne, France) were included in the longitudinal study. Animals were further divided into two groups according to the engrafted cells: PI group $(n=6)$ and INS-1E cell group $(n=5)$. After collection in an insulin syringe (mixed with 100-200 $\mu \mathrm{l}$ medium), either pre-labeled and transduced PIs $(n=200)$ or INS-1E cells $\left(n=10^{6}\right)$ were subcutaneously engrafted at the right thigh of the animals. Control injections were performed on the left side for all animals $\left(200 \mu \mathrm{l}\right.$ PBS for animals receiving PIs and $10^{6}$ unlabeled, transduced INS-1E cells for animals receiving INS-1E cells). During the transplantation procedure and the subsequent imaging sessions, animals were anesthetized with a ketamine $1000(100 \mathrm{mg} / \mathrm{ml}$, CEVA, Brussels, Belgium)/domitor (1 mg/ml, Janssen Pharmaceutica, Beerse, Belgium)/saline cocktail via intra-peritoneal injection. The following time points were used for imaging the animals of the two groups: PI group: day $0,1,3,7,14,28,42$, and 70 and INS-1E cell group: day $0,1,3,5,7,10,14$, and 21 . For PI group animals, single animals were sacrificed for confirmation by histology at day $1,3,7,21$, and 70 .

\section{In vivo BLI, FLI, and F-19 MRI}

For in vivo imaging, the same instruments (IVIS Spectrum and 9.4 Bruker MRI) were used for 
multimodal imaging as for the in vitro experiments. Prior to in vivo BLI sessions, D-luciferin, dissolved in PBS $(15 \mathrm{mg} / \mathrm{ml})$, was injected intravenously $(126 \mathrm{mg} / \mathrm{kg}$ body weight). All in vivo optical images were acquired using the same protocols and settings as for the in vitro experiments.

For both H-1 and F-19 MRI, animals were fixed using the manufacturer's animal holder (Bruker BioSpin). The tails of animals were left outside of the coil to facilitate the injection of anesthetics using a pre-fixed infusion line with a 27-gauge butterfly needle. Anatomical H-1 MR images were acquired using a rapid spin echo (RARE) sequence (for more details see ESM). F-19 MRI was performed using similar parameters as for the H-1 MRI with the following modifications $(\mathrm{TR}=1 \mathrm{~s}$; $\mathrm{TE}=$ $15.9 \mathrm{~ms}$; number of averages $=250$; number of slices $=$ 10; slice thickness $=2.5 \mathrm{~mm}$; matrix size $=100 \times 50$; acquisition time $\sim 30 \mathrm{~min}$ ). Orientation and dimension of the slice packages was kept identical for $\mathrm{H}-1$ and F19 MRI to facilitate co-registration. All MRI measurements were respiration-gated.

\section{Histology}

Animals that underwent PI transplantation were sacrificed at different time points (day 1, 3, 7, 21, and 70) and perfused with $4 \%$ paraformaldehyde for fixation. Right femoral tissue from the PI transplantation was collected for performing insulin staining (see ESM).

\section{Image Processing}

All reported BLI and FLI images were superimposed by a gray-scale photographic image with anatomical information and a pseudo-color image with functional information (see ESM). MRI data was processed using the ImageJ software (NIH, USA) (see ESM). For the longitudinal study, each animal's quantified BLI, FLI, and F-19 MR signals from the different time points were normalized to the data from the corresponding first time point.

\section{Statistical Analysis}

All statistical analyses and linear regressions were performed using the Prism 6 software (GraphPad Software, CA, USA). Either an ANOVA test with a Bonferroni post-test for multiple groups' comparisons or a Student's $t$ test for two group comparisons was used to determine statistical differences between different experimental groups. $P$ values less than 0.05 were considered significant.

\section{Results}

\section{In vitro Characterization of Labeled and Transduced INS-1E Cells}

Initial experiments were performed with the INS-1E cell line because of their beta cell-like properties and easy handling. First, we generated a stable firefly luciferase expressing INS$1 \mathrm{E}$ cell line using a multicistronic LV carrying the hEF1 $\alpha$ promoter to drive 3 flagFluc and PuroR for selection of transduced cells after adding puromycin. This resulted in a transduction rate of over $90 \%$.

By varying the particle concentration (final fluorine concentration: $10-50 \mathrm{mM}$ ) and labeling time (4 and $24 \mathrm{~h}$ ), PFCE labeling conditions were optimized to achieve sufficient PFCE uptake while retaining cell viability and function. A relatively long labeling time was necessary to improve the intracellular uptake as shown in Fig. 1a. Using the same PFCE concentration, 24-h labeling resulted in significant higher fluorine concentration per cell when compared with a 4-h labeling. Unexpectedly, no significant differences in uptake were seen for concentrations between 10 and $30 \mathrm{mM}$, while a significantly higher uptake of PFCEs was seen for a concentration of $50 \mathrm{mM}$. Cell viability, relative to unlabeled cells, was assessed for the different concentrations after labeling for $24 \mathrm{~h}$ (Fig. 1b). Although no significant difference was found between the control group and PFCE-labeled INS-1E cells, high concentrations (e.g., $50 \mathrm{mM}$ ) showed a marginally lower number of viable cells. This might be an implicit reflection of adverse effects on the cellular doubling time, considering the relatively long doubling times of INS-1E cells. Based on previous work on the required intracellular fluorine concentrations for detection by F-19 MRI and to avoid any adverse effects on cell biology, we established a concentration of $20 \mathrm{mM}$ fluorine and a labeling time of $24 \mathrm{~h}$ for pre-labeling INS-1E cells with PFCE in subsequent experiments [26]. This results in an average of $3.7 \times 10^{12}$ fluorine atoms per cell.

In addition, the stability of the fluorine label inside the cells was also tested by prolonging the culture time of prelabeled cells in the medium in absence of any PFCE contrast agent for another $24 \mathrm{~h}$. As shown in Fig. 1c, no significant change of intracellular fluorine content was found. Furthermore, we were also not able to detect F-19 NMR signal from the supernatant of the culture medium with our routine F-19 NMR spectroscopy protocol.

For illustration purposes, in vitro BLI, FLI, and F-19 MR images of labeled, transduced INS-1E cells are shown in Fig. 1df. In contrast to the negative controls (untreated cells and PBS), PFCE-labeled and transduced INS-1E cells $\left(3 \times 10^{5}\right.$ cells seeded in a six-well plate resulting in a few hundred cells/ $\mathrm{mm}^{3}$ ) were detectable by both BLI and FLI (Fig. 1d, e). A linear correlation between signal intensity and viable cells was found for BLI (see ESM). Furthermore, within an acquisition time of approximately $30 \mathrm{~min}, 5 \times 10^{5}$ INS-1E cells $(\sim 4000-5000$ cells/ pixel) could be detected by F-19 MRI with a signal-to-noise ratio (SNR) of about 
a

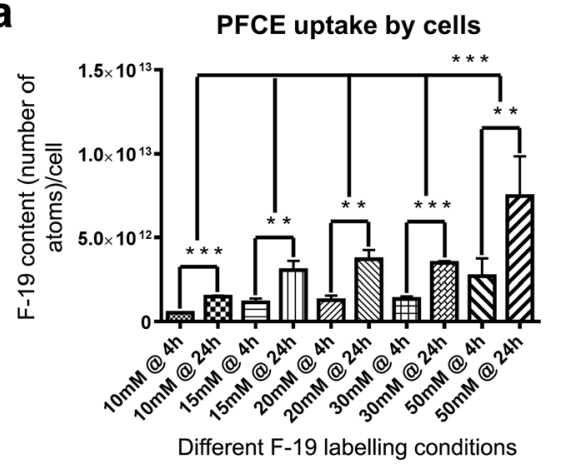

d

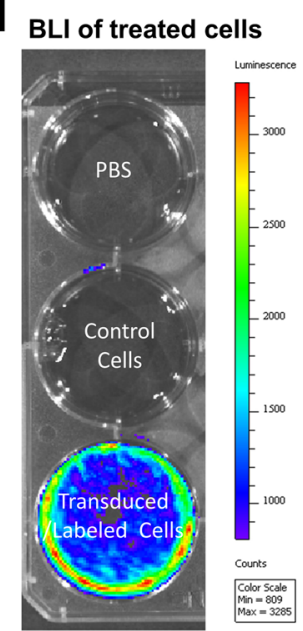

b

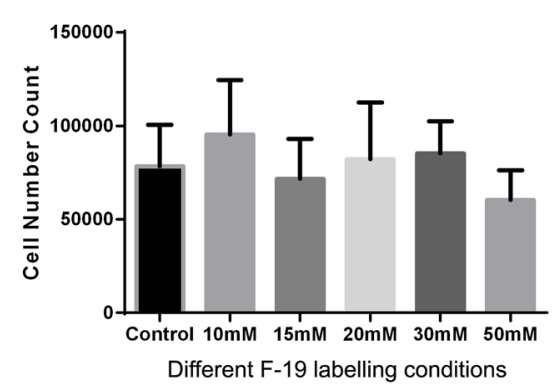

e

e

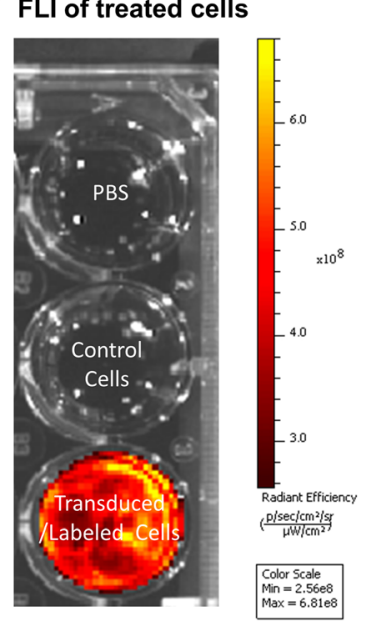

C

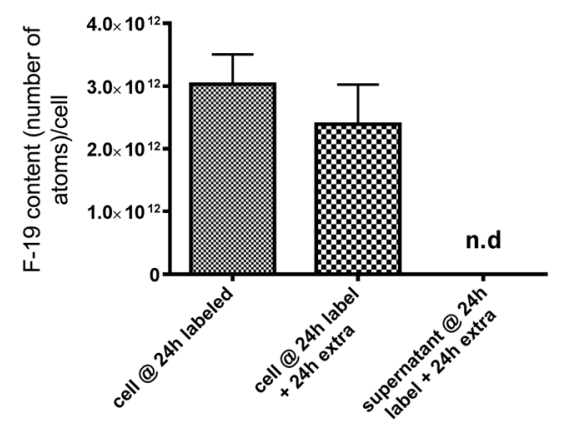

f

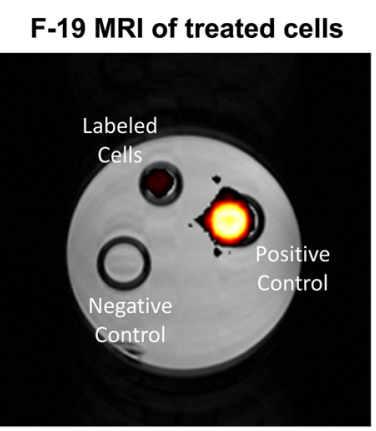

Fig. 1 In vitro characterization of labeled and transduced INS-1E cells (error bars: SD). a PFCE nanoparticle uptake by INS-1E cells based on F-19 NMR spectroscopy and expressed as fluorine content (number of atoms) per cell. Fluorine uptake was determined for the different labeling conditions (concentration:10, 15, 20, 30, and $50 \mathrm{mM}$; time: 4 and $24 \mathrm{~h}$ ). Significant differences were found between 4 and $24 \mathrm{~h}$ at different concentrations $(p<0.001$ at $10 \mathrm{mM}, p=0.0055$ at $15 \mathrm{mM}, p=0.0025$ at $20 \mathrm{mM}, p<0.001$ at $30 \mathrm{mM}, p=0.0036$ at $50 \mathrm{mM}$ ), significant difference found between $50 \mathrm{mM}$ with other concentrations at both 4 and $24 \mathrm{~h}(\boldsymbol{p}<0.001)$. b Cell viability determined after exposing INS-1E cells for $24 \mathrm{~h}$ to different concentrations of PFCE particles (fluorine concentration: 10,15, 20,30, and $50 \mathrm{mM}$ ) for $24 \mathrm{~h}$ (no significant difference found). c Assessment of PFCE released by labeled INS-1E cells. The fluorine content of PFCE-labeled cells $(24 \mathrm{~h}$ labeled with $20 \mathrm{mM}$ final fluorine concentration) was assessed after labeling and after extra $24 \mathrm{~h}$ incubation in PFCE (fluorine)-free medium. In addition, the supernatant from the latter cells were also collected for F-19 NMR spectroscopy (no significant difference found). d Representative BLI images (rainbow scale) of treated INS-1E cells overlaid with background photo (top: PBS; middle: untransduced control cells; bottom: $3 \times 10^{5} \mathrm{PFCE}-$ labeled cells that were transduced with a LV carrying the hEFla promoter, the 3flagFluc and a PuroR gene). e Representative FLI (hot scale, excitation wavelength: $648 \mathrm{~nm}$ and emission wavelength: $670 \mathrm{~nm}$ ) of treated INS-1E cells overlaid with background photo (top: PBS; middle: unlabeled control cells; bottom: $3 \times 10^{5}$ PFCElabeled (24 h labeling with $20 \mathrm{mM}$ fluorine concentration) and transduced cells). $\mathbf{f}$ Representative F-19 MR image (hot scale) of a phantom consisting of Eppendorf tubes in agar. The F-19 MR image is overlaid with its proton MR image (gray scaled). The respective Eppendorf tubes contain $5 \times 10^{5}$ unlabeled INS-1E cells (negative control), $5 \times 10^{5}$ labeled INS-1E cells, and PFCE particles (25 mM fluorine, positive control).

8 (Fig. 1f), which indicates a detection limit of a few thousands cells/pixel.

\section{In vitro Characterization of Labeled and Transduced Pancreatic Islets}

Considering the more complex composition of PIs, which consist of 1000 to 2000 endocrine cells and the fact that isolated PIs cannot be maintained for a long time in culture, a modified strategy was needed for labeling and transducing PIs. Using the same protocol as for the INS-1E cell transduction, luciferase expression by PIs failed almost completely as also shown in the in vitro BLI experiments (Fig. 2a). Prolongation of the incubation time from 24 to $48 \mathrm{~h}$ resulted in successful PI transduction and successful detection by BLI.

The same PFCE labeling procedure as used for the INS1E cells could be used to label PIs with PFCE. Maximum 
a

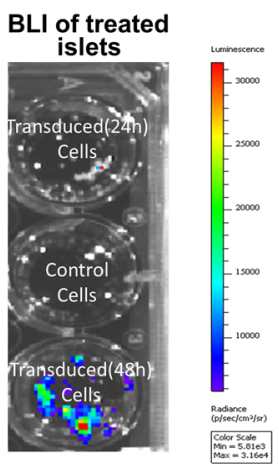

b

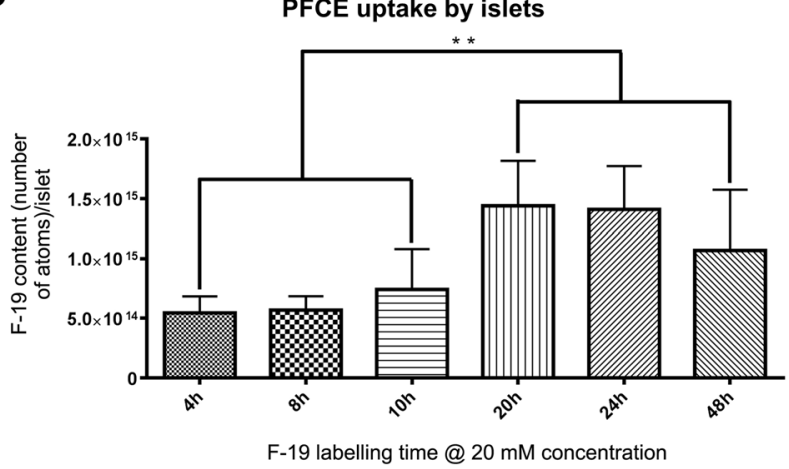

C

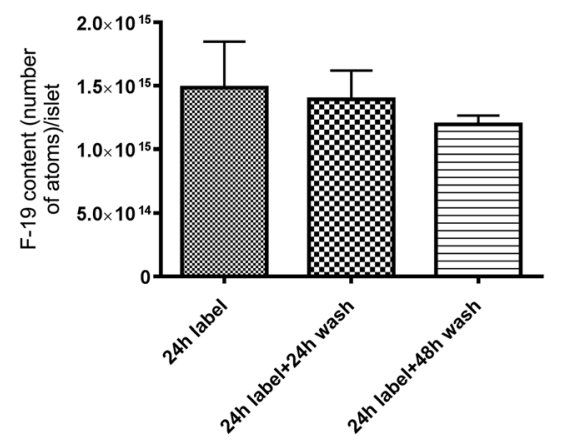

d

Microscopic images of treated islets
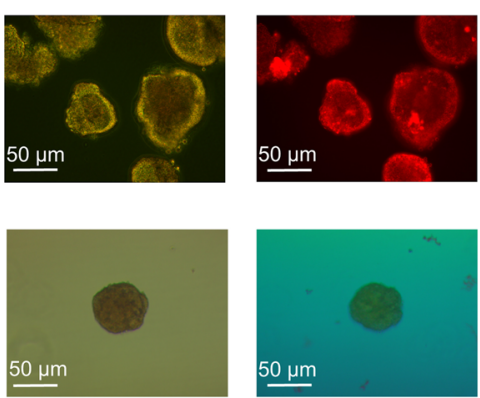

e

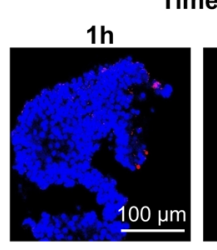

Time series of confocal microscopic image of treated islets $8 \mathrm{~h}$
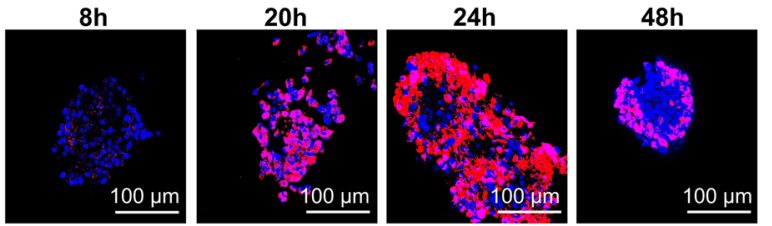

Fig. 2 In vitro characterization of PFCE-labeled and transduced pancreatic islets (error bar indicates standard deviation). a Representative BLI image (rainbow scale) of a LV carrying the hEFla promoter to drive 3flagFluc and a PuroR gene transduced Pls overlaid with background photo (top: 50 Pls with $24 \mathrm{~h}$ transduction; middle: 50 controlled Pls; bottom: 50 Pls with $48 \mathrm{~h}$ transduction). $\mathbf{b}$ Determination of PFCE particle uptake (final fluorine concentration: $20 \mathrm{mM}$ ) by Pls after increasing incubation times $(4,8,10,20,24$, and $48 \mathrm{~h}$ ) using F-19 NMR spectroscopy (significant difference between short incubation times $(4,8$, and $10 \mathrm{~h}$ ) with long incubation times $(20,24$, and $48 \mathrm{~h}), p=0.0071)$. c Assessment of PFCE released by pancreatic islets (error bar: standard deviation). The fluorine content of labeled PIs ( $24 \mathrm{~h}$ labeling with $20 \mathrm{mM}$ final fluorine concentration) were compared with islets that underwent the same labeling procedure with an extra washing step and 24-/48-h incubation in PFCE/ fluorinefree medium (no significant difference found). $\mathbf{d}$ Representative microscopy images of both labeled and transduced pancreatic islets (top/bottom left: light microscopy images of Pls; top right: fluorescence microscopy image of Pls; bottom right: fluorescence microscopy of Pls after trypan blue staining). Scale bar, $50 \mu \mathrm{m}$. e Time series of confocal microscopy of labeled pancreatic islets (blue: nuclei counterstained with Hoechst dye; red: PFCE particles containing DiD dye; pink: co-localization of nuclei and PFCE particles). From left to right: representative islets at different time points after incubation with PFCE particles $(1,8,20,24$, and $48 \mathrm{~h})$. Scale bar, $100 \mu \mathrm{m}$.

uptake was achieved after 20 to $24 \mathrm{~h}$ of exposure to PFCE resulting in a total fluorine content of $20 \mathrm{mM}$. On average, each islet contained approximately $1.44 \times 10^{15}$ fluorine atoms (Fig. 2b). The release study confirmed that there was no significant leakage of PFCE label from the PIs even after $48 \mathrm{~h}$ in fluorine-free medium (Fig. 2c). Since the cells of isolated PI do not proliferate as the INS-1E cells, it can be expected that the sustainability of the fluorine label in PIs would be longer than for the cell line. Hence, the final procedure for PI labeling consisted of an initial 24-h exposure of PIs to LV ( $5 \mu 1$ per well) and a subsequent addition of PFCE particles $(20 \mathrm{mM})$ to the LV containing medium with an exposure for another $24 \mathrm{~h}$. Hereby, we were able to simultaneously label and transduce PIs.

By labeling the lipid shell of the PFCE containing particles with the red DiD fluorescent dye, uptake and internalization of the PFCE particles by PIs could be confirmed by using fluorescence microscopy (Fig. 2d). Trypan blue staining of treated PIs confirmed the viability of the intact PIs (Fig. 2d). The insulin content of control PIs $(898 \pm 50 \mu \mathrm{U} /$ islet $)$ and treated PIs $(972 \pm 38 \mu \mathrm{U} /$ islet $)$ was similar, suggesting an intact capacity of PIs for insulin production. Results obtained from the GSIS test showed that the secretory responsiveness to the hexose is preserved in both control and transduced PIs. The insulin secretion increased from $56 \pm \mu \mathrm{U} /$ islet $(n=3$, glucose $2.8 \mathrm{mM})$ to $310 \pm 89 \mu \mathrm{U} /$ islet $(n=5$, glucose $16.7 \mathrm{mM})$ in the control group. In the transduced PIs, the insulin secretion increased from $50 \pm 3 \mu \mathrm{U} /$ islet $(n=3$, glucose $2.8 \mathrm{mM})$ to $192 \pm$ $94 \mu \mathrm{U} /$ islet $(n=5$, glucose $16.7 \mathrm{mM})$.

To further confirm localization of PFCE particles within the islets, a series of confocal microscopy images of labeled 
PIs were acquired after exposure to PFCE particles for 1 to $48 \mathrm{~h}$ (Fig. 2e). Particles were found both inside the cells and in the interstitial space of PIs. The different fluorescence signal intensities for the different PFCE labeling experiments further confirmed our findings that the optimal labeling time should be between 20 and $24 \mathrm{~h}$. Z-stack slicing of individual cells (see ESM) further indicated a notable uptake of PFCE nanoparticles in the cells of PIs after a 24-h incubation time.

For bioluminescence imaging, several sub-regions of the well plate showed detectable BLI signal if at least 50 islets were plated, which indicates a detection limit of a few islets by in vitro BLI (Fig. 2a). For fluorescence imaging, the high signal intensity against background showed a similar detection limit as for BLI (ESM). Using F-19 MRI, ten islets per pixel were detectable within an acquisition time of 30 min (SNR 12, see ESM).

\section{Multimodal In vivo Imaging of Transplanted INS- IE Cells}

Multimodal in vivo imaging of subcutaneously transplanted INS$1 \mathrm{E}$ cells was performed by grafting one million unlabeled but transduced (fLuc) INS-1E cells as well as PFCE-labeled and transduced (fLuc) INS-1E cells subcutaneously at the left and right thigh of the same animal, respectively. As expected, neither F-19 MRI signal nor FLI signal could be detected from the unlabeled and untransduced cells (Fig. 3a, c). Both FLI and F-19 MRI sustained detectable signal for PFCE-labeled cells over a period of 21 days. A significant drop in signal intensity was observed over time (Fig. 3b, d). The FLI signal of labeled cells was gradually reduced to $53 \pm 4.6 \%$ of its original intensity after implantation. A similar decrease to $68 \pm 4.1 \%$ of its initial value was also observed for the F-19 MRI. A linear correlation between the intensity of F-19 MRI and FLI signal intensity was noticed (see Fig. 3e).

In contrast to external contrast agents, BLI provides direct information on the viability of transplanted cells (Fig. 3f), which results in a considerably larger variability between different animals. As shown in Fig. 3g, due to the harsh environment (limited oxygen and nutrition supply) at the transplantation site and a potential immune response mediated by the engrafted cells, a rapid loss in BLI signal intensity (reduction by $70 \%$ within 1 day after transplantation) and hereby of viable cells was observed. Until 3 days after engraftment, only about $14 \pm 15.5 \%$ of cells remained viable. From this time point on, the BLI signal intensity and number of cells started to increase again ( $81 \%$ from day 3 to day 5 and from day 5 to day 7), most likely due to cell proliferation. However, the time period between day 7 and day 10 seemed to be critical, as only one animal out of three showed remaining detectable BLI signal present on day 10 with a further $62.4 \%$ increase in cell number (signal intensity) until day 21 . Besides the increase in BLI signal intensity, no formation of a (tumor) mass was seen in the $\mathrm{H}$ 1 MRI.

\section{Multimodal In vivo Imaging of Transplanted Pancreatic Islets}

Longitudinal in vivo tracking of transplanted PIs was carried out after transplanting 200 transduced, PFCE-labeled PIs to the right thigh. PBS injection served as a negative control on the left thigh. Compared to INS-1E cell engraftment, PIs were monitored for a longer period (up to 70 days after engraftment. Similar to the temporal profile of transplanted INS-1E cells, both FLI and F-19 MRI signal intensity decreased over time. A similar linear correlation between the two methods was observed (Fig. 4a-e). More interestingly, after the last time point at day 70 , there was still $47 \%$ of F-19 MRI signal and $9 \%$ FLI signal intensity remaining. This provides evidence that labeling with PFCE particles is suitable for longitudinal cell tracking for up to several months. The larger drop in FLI signal intensity when compared to the F-19 MRI signal intensity is most likely due to the degradation of the fluorescent dye.

For BLI, the signal intensity showed a dramatic drop on the first day (Fig. 4f, g). Starting from day 14, there was no signal detectable by BLI for all animals. This indicates that the number of remaining viable islets was below the detection limit of BLI. We were able to confirm by immunohistochemistry (anti-insulin labeling using avidin-biotin-peroxidase complex with diaminobenzidine, see Fig. 4h) that viable, insulin-producing PIs were still present after day 7 and even after day 21 (in the latter case with considerably degraded PI morphology). This indicates that most likely the transduced cells of PIs at the outer rim of the islets started to die first, while some untransduced cells remained functional.

\section{Discussion}

Thanks to negligible background signal, F-19 MRI has found its position in cellular imaging [31]. Together with high-resolution H-1 MRI, which provides an anatomical reference, F-19 MRI can offer accurate information regarding the location of transplanted cells in vivo and the possibility of direct quantification. Among different fluorinated contrast agents described in the literature [32, 33], about two thirds are perfluocarbon (PFC) based. A remarkable strength of PFC is its often high payload of fluorine atoms per molecule. PFCEs used in this study contain 20 equivalent fluorine atoms, which exhibits one single resonance peak in its F-19 NMR spectrum. By using positively charged PFCE particles, we showed for INS-1E cells and PIs can be labeled with fluorinated particles without using transfection agents or targeting moieties. The average fluorine atom uptake per INS$1 \mathrm{E}$ cell or islet cell was about 1.5 to $4 \times 10^{12}$, which is comparable with the typical range of cellular fluorine label required for F-19 MRI-based cell tracking $\left(10^{11} \sim 10^{13}\right)$ [27, 34]. Using our experimental setup, $10^{16}$ fluorine spins per voxels are required to achieve sufficient SNR, which results in a detection limit of several thousand cells or ten PIs. PFCE particles are biologically inert, resulting in no or only limited impact on cell phenotypes and function $[15,32]$. This has been confirmed in our study where no adverse effects of the fluorine label on cell viability and functionality was observed. However, the side effects on cells 
a

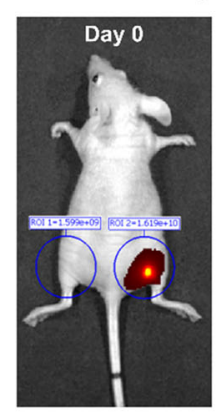

Representative in vivo FLI of transplanted cells
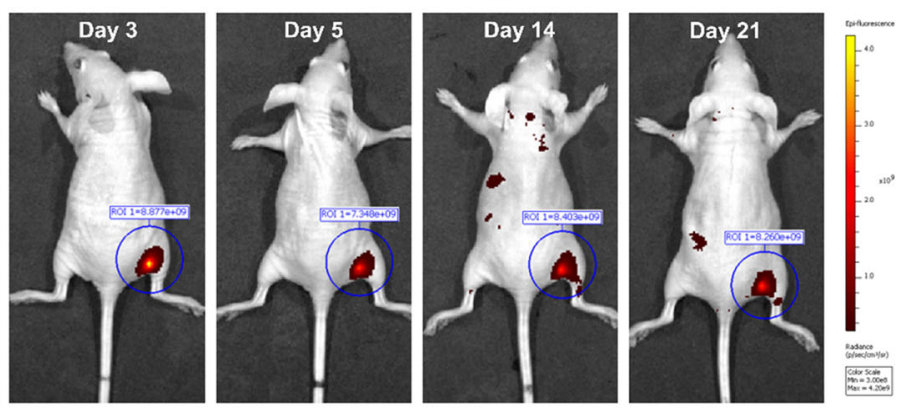

C

\section{Representative in vivo F-19 MRI of transplanted cells}
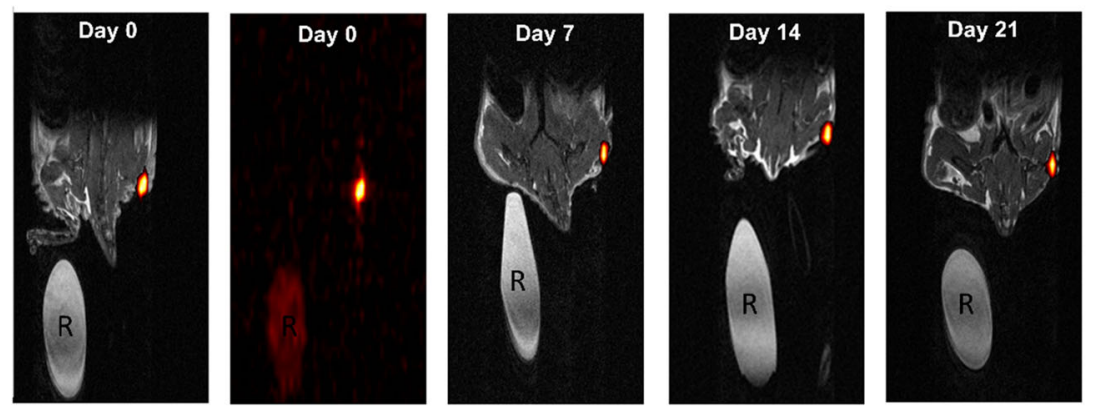

f

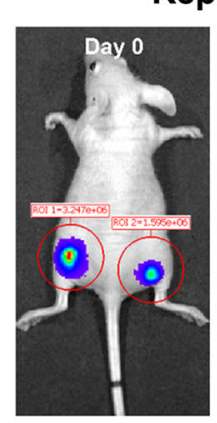

Representative in vivo BLI of transplanted cells
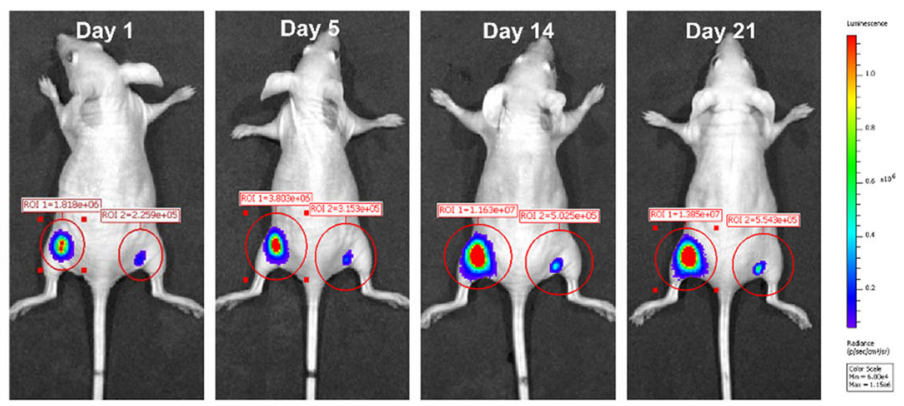

b

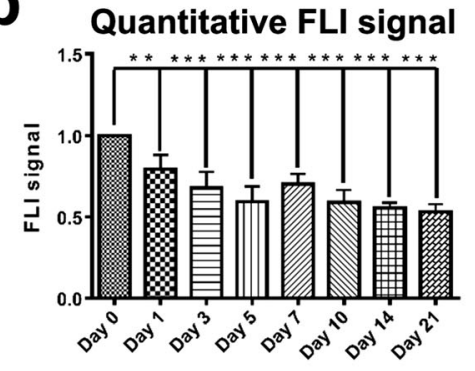

d

Quantitative F-19 MRI signal

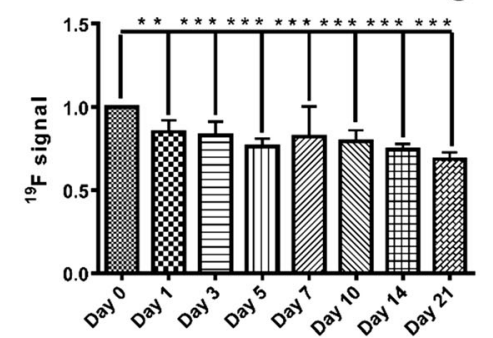

e

\section{Correlation between FLI} and MRI signals

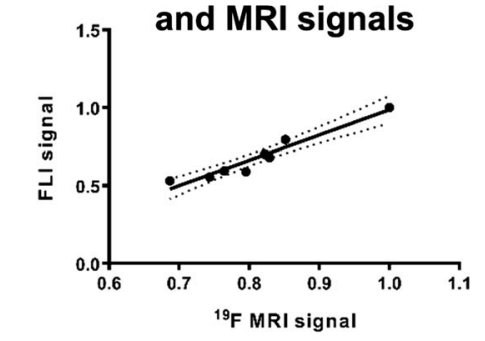

g

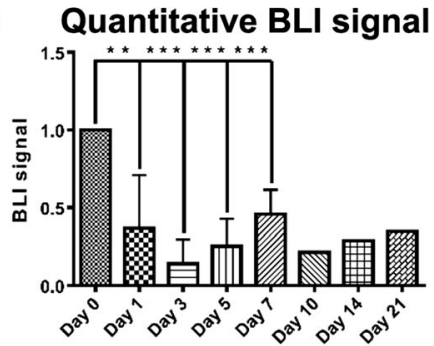

Fig. 3 Multimodal in vivo imaging of transplanted INS-1E cells $\left(10^{6}\right.$ cells suspended in 100-200 $\mu$ l medium were injected at both the right (labeled and transduced) and left (only transduced) thigh, $n=6$, Swiss nude mice, female, 7-9 weeks old) (error bar indicates standard deviation). a Representative in vivo FLI images (hot scaled) of transplanted INS-1E cells overlaid with background photographic images at different time points after engraftment (from left to right: day 0 , day 3 , day 5, day 14 , and day 21). b Quantification of in vivo FLI signal intensity of transplanted INS-1E cells over 21 days (significant difference found between all time points to day $0, p=0.007$ for day 1 and $p<0.001$ for all other time points). c Representative in vivo F-19 MR images (hot scale) of INS-1E cells overlaid with anatomical H-1 MR image (gray scaled) at different time points (from left to right: day 0 , day 0 (only F-19 MRI without anatomical background), day 7 , day 14 , and day 21 ). $R$ represents a reference containing $15 \mathrm{mM}$ (fluorine) of PFCE particles. d Quantification of in vivo F-19 MRI signal intensity of engrafted INS-1E cells over 21 days. For quantification purposes, regions-of-interest from the site of engraftment were compared with respective regions in the Eppendorf tube containing $15 \mathrm{mM}$ (fluorine) of PFCE particles as a reference (significant difference found between all time points to day $0, p=0.0014$ for day 1 and $p<0.001$ for all other time points). e Correlation between in vivo FLI and F-19 MRI signal intensity of transplanted INS-1E cells over a time period of 21 days $(\mathrm{Y}(\mathrm{FLI}$ signal $)=1.621 \times \mathrm{X}(\mathrm{F}-19$ signal $)-0.6345, R=$ 0.9671). f Representative in vivo BLI (rainbow scale) of transplanted INS-1E cells overlaid with background photo at different time points (left $\rightarrow$ right: day 0 , day 1 , day 5 , day 14 , and day 21 ). $\mathbf{g}$ Quantification of in vivo BLI signal intensity of the region containing transplanted INS-1E cells over a time period of 21 days (significant difference found between day $1,3,5$, and 7 to day $0, p=0.0032$ for day 1 and $p<0.001$ for other time points). 
a

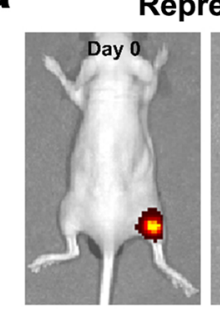

Rep
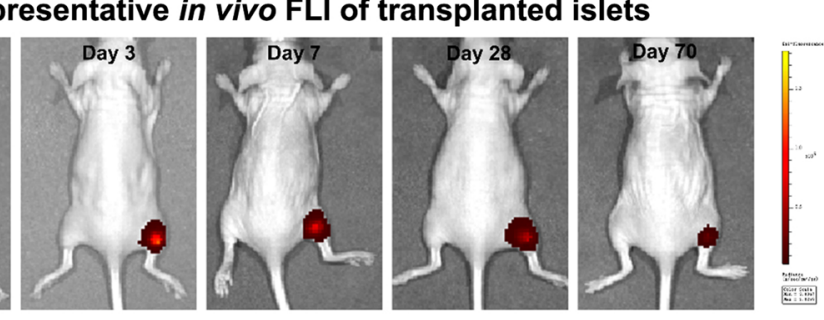

b

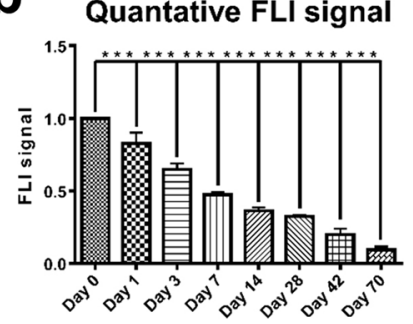

\section{Representative in vivo F-19 MRI of transplanted islets}
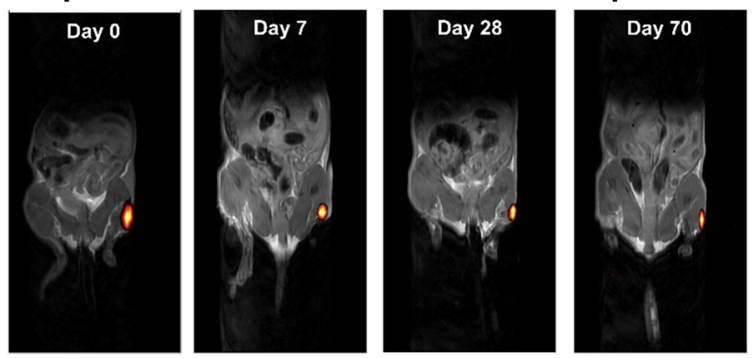

f

Representative in vivo BLI of transplanted cells
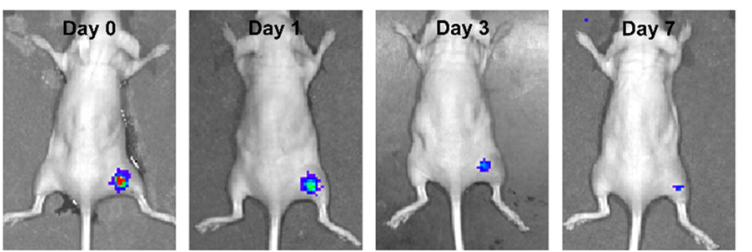

d

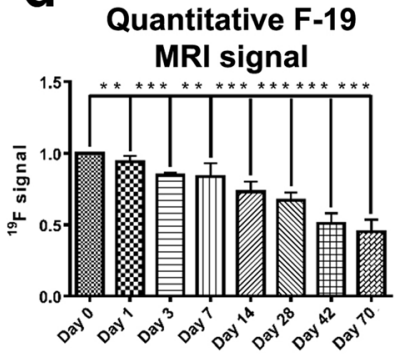

e

Correlation between FLI and F-19 MRI signal

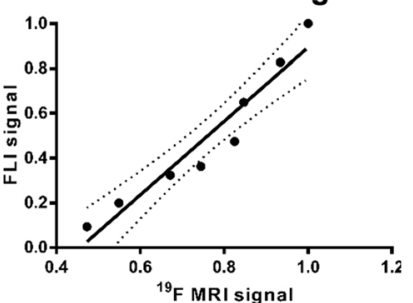

9 Quantative BLI signal

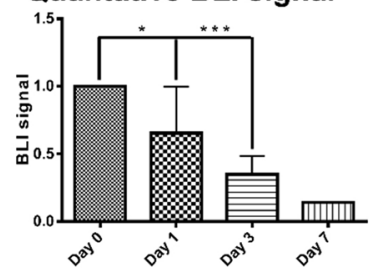

h

Histology of transplanted islets using anti-insulin antibodies
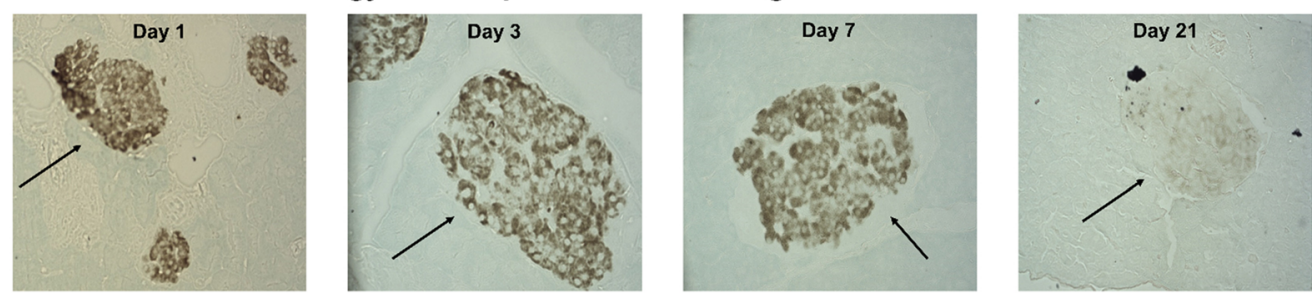

Fig. 4 Multimodal in vivo imaging of transplanted pancreatic islets (200 Pls (labeled and transduced) mixed with $100-200 \mu \mathrm{l}$ medium at right thigh and $200 \mu \mathrm{l}$ PBS at left thigh, $n=6$, Swiss nude mice (female, 7-9 weeks old)). a Representative in vivo FLI images (hot scaled) of transplanted pancreatic islets overlaid with background photo at different time points (left $\rightarrow$ right: day 0 , day 3 , day 7 , day 28 , and day 70 ). b Quantitative FLI signal of in vivo tracking transplanted pancreatic islets over 70 days (significant difference found between all time points to day $0, p<0.001$ ). c Representative in vivo $\mathrm{F}-19 \mathrm{MRI}$ signal (hot scale) of transplanted pancreatic islets overlaid with overlaid with proton image (gray scaled) at different time points (left $\rightarrow$ right: day 0 , day 7, day 28, and day 70). d Quantitative F-19 signal of in vivo tracking transplanted pancreatic islets over 70 days (significant difference found between all time points to day $0, p=0.0054$ for day 1 and $p<0.001$ for all other time points). e Correlation between FLI signal with F-19 signal of in vivo tracking transplanted pancreatic islets over 70 days $(\mathrm{Y}(\mathrm{FLI}$ signal) $=1.647 \times \mathrm{X}$ (F-19 signal) $-0.7528, R=0.9628)$. f Representative in vivo BLI images (rainbow scaled) of transplanted pancreatic islets overlaid with background photo at different time points (left $\rightarrow$ right: day 0, day 1, day 3, and day 7). g Quantitative BLI signal of in vivo tracking transplanted pancreatic islets over 70 days (significant difference found between day 1 to day $0, p=0.0347$ and day 3 to day $0, p<0.001$ ). $\mathbf{h}$ Light microscopy of pancreatic islets using Guinea pig anti-insulin antibodies (left $\rightarrow$ right: day 1 , day 3 , day 7 , day 21 ). Arrow points to labeled Pls.

are known to be strongly dose-dependent [35]. With the highest fluorine concentration in culture medium $(50 \mathrm{mM})$, the reduction of cellular viability based on cell density could be expected. This is not surprising according to a previous study [36], where an effect of fluorine particle labeling on the migratory and proliferation capacity on neural stem cells was found.

Incorporation of a fluorescent dye into PFC emulsions has been described before, which allows confirmation of cell labeling 
by fluorescence microscopy, flow cytometry, and in vivo optical imaging [37]. Similarly, radiotracer-based in vivo imaging approaches have been combined with optical validation ex vivo [38]. We have used a lipophilic membrane-bound red fluorescent DiD dye in our PFCEs, which can be integrated rapidly and in a stable manner with long retention and limited toxicity [39]. The internalization of PFCE particles into cells was hereby confirmed even several months after PI engraftment. The red-shift of the DiD dye also allowed reliable in vivo detection, resulting in a linear correlation between in vivo F-19 MRI and FLI signal intensities of transplanted INS-1E cells and PIs, which is in agreement with another similar reagent [34]. As the PFC emulsions were stable inside the INS-1E cells/PIs, both F-19 MRI and FLI allow quantification of cell/PI numbers in vivo after in vitro precalibration and under the assumption that cell labeling is uniform. However, translation to the in vivo situation is not straightforward, where cell division, phagocytosis of dead cells, or released labels could lead to misinterpretation.

One limitation of MRI and FLI is the fact that direct assessment of the cell viability is not possible. In contrast, in vivo BLI of genetically modified INS-1E cells and PIs to express fLuc can provide semi-quantitative information on cell viability, as the generation of bioluminescence by fLuc expressing cells requires ATP and oxygen. As shown before, genetically engineered cells and PIs did show survival rates and cell functionality comparable to wild-type cells/PIs [40]. In vivo monitoring of INS-1E cells and PIs by using BLI identified early onset of some substantial graft rejection (almost immediately after the transplantation), while both F-19 MRI and FLI showed delayed response, which underlies the importance of multimodal imaging approaches for cross-validation and proper image interpretation.

The selected subcutaneous transplantation site is an attractive location for cell/PI tracking since the related transplantation procedure is minimally invasive. From an imaging point of view, the superficial location of the subcutaneous space is perfectly suited for performing optical imaging due to only marginal light scattering. For conventional transplantation sites like intraportal engraftment into the liver, true 3D imaging methods like MRI or PET are needed. For similar reasons, optical imaging methods are less suitable for translation to the clinic. Radionuclide imaging like PET and SPECT have been used for targeting beta cells (for example by targeting HTP/ GLP-1 analogs) [41, 42]. PET and SPECT are in particular relevant for the assessment of intrinsic beta cell mass and beta cell function. The relatively short half-life of PET tracers limits its application [20, 29]. For monitoring engrafted islets/beta cells independent of remaining intrinsic beta cells, transfected/ transduced cells for reporter gene-based approaches were suggested [20, 29].

As shown by our data, another downside of subcutaneous transplantation is the poor survival rate of transplanted cells/ PIs due to a potentially evoked immune response together with hypoxia and a hypo-vascular environment [43]. As such, the minimum number of islets required for recovering diabetic animal models are reported to be 800 for mice and 5000 for rats $[44,45]$. Improved outcome can be achieved by induction of angiogenesis/pre-vascularization using biodegradable foreign material such as biodegradable PGA polymers [46] and stainless steel mesh [47] or scaffolds made of extracellular matrices [48]. Other novel methods, such as encapsulation of PIs for immune isolation [49], cotransplanted PIs with adipose and bone marrow stem cells for vascularization [50] are also potentially beneficial.

In summary, we optimized the transduction and labeling protocols for both PIs and INS-1E cells, which confirmed internalization of PFCE particles and expression of firefly luciferase. No significant side effects on cell viability and function were noticed for the conditions used in this study, making this a safe approach. Our data suggested F-19 MRI is suitable for highresolution localization of transplanted cells and PIs; FLI is essential for confirmation of contrast agent localization by histology; and BLI is a reliable method to assess cell viability and survival after transplantation. This imaging platform is not only limited to subcutaneous transplantation but can also be applied to compare different transplantation sites, such as intramuscular, intraportal liver injection, into the omentum, into the anterior chamber of the eye, and others [9].

\section{Conclusions}

We propose a tri-modal imaging platform by combining BLI, FLI, and F-19 MRI for tracking subcutaneously transplanted PIs or INS-1E cells for the in vivo characterization of experimental models for islet transplantation. With this imaging approach, novel strategies for improving outcome of PI transplantation could be better evaluated and compared in longitudinal studies, reducing the number of animals needed and reducing statistical variability when compared with studies entirely relying on histology at particular time points.

Acknowledgments. We thank Mrs. Tinne Buelens, Mrs. Amy Hillen, and Mrs. Ann Van Santvoort for technical support.

Funding This work was supported by grants from the Flemish Government FWO project G.0A75.14 and G.0B28.14, from the Agency for Innovation by Science and Technology (IWT 130065 (SBO MIRIAD) and IWT 140061 (SBO NanoCoMIT)), from the European Commission (FP7-MC-ITN Betatrain (289932) and FP7NMP VIBRANT (228933)), and from the KU Leuven program financing IMIR (PF 2010/017).

Compliance with Ethical Standards

Conflict of Interest

The authors declare that they have no conflict of interest.

\section{References}

1. Alberti KG, Zimmet PZ (1998) Definition, diagnosis and classification of diabetes mellitus and its complications. Part 1: diagnosis and classification of diabetes mellitus provisional report of a WHO consultation. Diabet Med 15:539-553

2. Diabetes C, Complications Trial Research G, Nathan DM et al (1993) The effect of intensive treatment of diabetes on the development and 
progression of long-term complications in insulin-dependent diabetes mellitus. N Engl J Med 329:977-986

3. Ballinger WF, Lacy PE (1972) Transplantation of intact pancreatic islets in rats. Surgery 72:175-186

4. Scharp DW, Lacy PE, Santiago JV, McCullough CS, Weide LG, Falqui L, Marchetti P, Gingerich RL, Jaffe AS, Cryer PE, Anderson CB, Flye MW (1990) Insulin independence after islet transplantation into type I diabetic patient. Diabetes 39:515-518

5. Shapiro AM, Pokrywczynska M, Ricordi C (2017) Clinical pancreatic islet transplantation. Nat Rev Endocrinol 13:268-277

6. Robertson RP (2004) Islet transplantation as a treatment for diabetes - a work in progress. N Engl J Med 350:694-705

7. Gruessner RW, Gruessner AC (2013) The current state of pancreas transplantation. Nat Rev Endocrinol 9:555-562

8. Farney AC, Sutherland DE, Opara EC (2016) Evolution of islet transplantation for the last 30 years. Pancreas 45:8-20

9. Rajab A (2010) Islet transplantation: alternative sites. Curr Diab Rep 10:332-337

10. Shapiro AM, Ricordi C, Hering BJ et al (2006) International trial of the Edmonton protocol for islet transplantation. $\mathrm{N}$ Engl $\mathrm{J}$ Med 355:1318-1330

11. Barton FB, Rickels MR, Alejandro R, Hering BJ, Wease S, Naziruddin B, Oberholzer J, Odorico JS, Garfinkel MR, Levy M, Pattou F, Berney T, Secchi A, Messinger S, Senior PA, Maffi P, Posselt A, Stock PG, Kaufman DB, Luo X, Kandeel F, Cagliero E, Turgeon NA, Witkowski P, Naji A, O'Connell PJ, Greenbaum C, Kudva YC, Brayman KL, Aull MJ, Larsen C, Kay TWH, Fernandez LA, Vantyghem MC, Bellin M, Shapiro AMJ (2012) Improvement in outcomes of clinical islet transplantation: 1999-2010. Diabetes Care 35:1436-1445

12. Speier S, Nyqvist D, Cabrera O, Yu J, Molano RD, Pileggi A, Moede T, Köhler M, Wilbertz J, Leibiger B, Ricordi C, Leibiger IB, Caicedo A, Berggren PO (2008) Noninvasive in vivo imaging of pancreatic islet cell biology. Nat Med 14:574-578

13. Sakata N, Aoki T, Yoshimatsu G, Tsuchiya H, Hata T, Katayose Y, Egawa S, Unno M (2014) Strategy for clinical setting in intramuscular and subcutaneous islet transplantation. Diabetes Metab Res Rev 30:1-10

14. Arifin DR, Bulte JW (2011) Imaging of pancreatic islet cells. Diabetes Metab Res Rev 27:761-766

15. Malaisse WJ, Louchami K, Sener A (2009) Noninvasive imaging of pancreatic beta cells. Nat Rev Endocrinol 5:394-400

16. Lu Y, Dang H, Middleton B, Zhang Z, Washburn L, CampbellThompson M, Atkinson MA, Gambhir SS, Tian J, Kaufman DL (2004) Bioluminescent monitoring of islet graft survival after transplantation. Mol Ther 9:428-435

17. Virostko J, Radhika A, Poffenberger G, Chen Z, Brissova M, Gilchrist J, Coleman B, Gannon M, Jansen ED, Powers AC (2010) Bioluminescence imaging in mouse models quantifies beta cell mass in the pancreas and after islet transplantation. Mol Imaging Biol 12:42-53

18. Eriksson O, Eich T, Sundin A, Tibell A, Tufveson G, Andersson H, Felldin M, Foss A, Kyllönen L, Langstrom B, Nilsson B, Korsgren O, Lundgren T (2009) Positron emission tomography in clinical islet transplantation. Am J Transplant 9:2816-2824

19. Crenier L, Courtois P, Malaisse WJ (2001) Uptake of tritiated Dmannoheptulose by liver, pancreatic exocrine and endocrine cells. Int J Mol Med 8:155-157

20. Kim SJ, Doudet DJ, Studenov AR, Nian C, Ruth TJ, Gambhir SS, McIntosh CHS (2006) Quantitative micro positron emission tomography (PET) imaging for the in vivo determination of pancreatic islet graft survival. Nat Med 12:1423-1428

21. Tai JH, Foster P, Rosales A, Feng B, Hasilo C, Martinez V, Ramadan S, Snir J, Melling CWJ, Dhanvantari S, Rutt B, White DJG (2006) Imaging islets labeled with magnetic nanoparticles at 1.5 Tesla. Diabetes 55:2931-2938

22. Evgenov NV, Medarova Z, Pratt J, Pantazopoulos P, Leyting S, Bonner-Weir S, Moore A (2006) In vivo imaging of immune rejection in transplanted pancreatic islets. Diabetes 55:2419-2428

23. Jirak D, Kriz J, Herynek V et al (2004) MRI of transplanted pancreatic islets. Magn Reson Med 52:1228-1233

24. Biancone L, Crich SG, Cantaluppi V, Romanazzi GM, Russo S, Scalabrino E, Esposito G, Figliolini F, Beltramo S, Perin PC, Segoloni GP, Aime S, Camussi G (2007) Magnetic resonance imaging of gadolinium-labeled pancreatic islets for experimental transplantation. NMR Biomed 20:40-48
25. Maillard E, Juszczak MT, Langlois A, Kleiss C, Sencier MC, Bietiger W, Sanchez-Dominguez M, Krafft MP, Johnson PRV, Pinget M, Sigrist S (2012) Perfluorocarbon emulsions prevent hypoxia of pancreatic beta-cells. Cell Transplant 21:657-669

26. Liang S, Louchami K, Kolster H, Jacobsen A, Zhang Y, Thimm J, Sener A, Thiem J, Malaisse W, Dresselaers T, Himmelreich U (2016) In vivo and ex vivo 19-fluorine magnetic resonance imaging and spectroscopy of beta-cells and pancreatic islets using GLUT-2 specific contrast agents. Contrast Media Mol Imaging 11:506-513

27. Srinivas M, Heerschap A, Ahrens ET, Figdor CG, IJM V (2010) ${ }^{19}$ F MRI for quantitative in vivo cell tracking. Trends Biotechnol 28:363-370

28. Dewitte H, Geers B, Liang S, Himmelreich U, Demeester J, de Smedt SC, Lentacker I (2013) Design and evaluation of theranostic perfluorocarbon particles for simultaneous antigen-loading and ${ }^{19} \mathrm{~F}-$ MRI tracking of dendritic cells. J Control Release 169:141-149

29. Wolfs E, Holvoet B, Gijsbers R, Casteels C, Roberts SJ, Struys T, Maris M, Ibrahimi A, Debyser Z, van Laere K, Verfaillie CM, Deroose CM (2014) Optimization of multimodal imaging of mesenchymal stem cells using the human sodium iodide symporter for PET and Cerenkov luminescence imaging. PLoS One 9:e94833

30. Lacy PE, Kostianovsky M (1967) Method for the isolation of intact islets of Langerhans from the rat pancreas. Diabetes 16:35-39

31. Ahrens ET, Flores R, Xu H, Morel PA (2005) In vivo imaging platform for tracking immunotherapeutic cells. Nat Biotechnol 23:983-987

32. Srinivas M, Boehm-Sturm P, Figdor CG, de Vries IJ, Hoehn M (2012) Labeling cells for in vivo tracking using (19)F MRI. Biomaterials 33:8830-8840

33. Ahrens ET, Zhong J (2013) In vivo MRI cell tracking using perfluorocarbon probes and fluorine-19 detection. NMR Biomed 26:860-871

34. Janjic JM, Srinivas M, Kadayakkara DK, Ahrens ET (2008) Selfdelivering nanoemulsions for dual fluorine-19 MRI and fluorescence detection. J Am Chem Soc 130:2832-2841

35. Lutz J, Kettemann M, Racz I, Noth U (1995) Several methods utilized for the assessment of biocompatibility of perfluorochemicals. Artif Cells Blood Substit Immobil Biotechnol 23:407-415

36. Boehm-Sturm P, Aswendt M, Minassian A, Michalk S, Mengler L, Adamczak J, Mezzanotte L, Löwik C, Hoehn M (2014) A multimodality platform to image stem cell graft survival in the naive and stroke-damaged mouse brain. Biomaterials 35:2218-2226

37. Hitchens TK, Ye Q, Eytan DF, Janjic JM, Ahrens ET, Ho C (2011) ${ }^{19} \mathrm{~F}$ MRI detection of acute allograft rejection with in vivo perfluorocarbon labeling of immune cells. Magn Reson Med 65:1144-1153

38. Eter WA, Parween S, Joosten L, Frielink C, Eriksson M, Brom M, Ahlgren U, Gotthardt M (2016) SPECT-OPT multimodal imaging enables accurate evaluation of radiotracers for beta-cell mass assessments. Sci Rep 6:24576

39. Yumoto K, Berry JE, Taichman RS, Shiozawa Y (2014) A novel method for monitoring tumor proliferation in vivo using fluorescent dye DiD. Cytometry A 85:548-555

40. Fowler M, Virostko J, Chen Z, Poffenberger G, Radhika A, Brissova M, Shiota M, Nicholson WE, Shi Y, Hirshberg B, Harlan DM, Jansen ED, Powers AC (2005) Assessment of pancreatic islet mass after islet transplantation using in vivo bioluminescence imaging. Transplantation 79:768-776

41. Brom M, Woliner-van der Weg W, Joosten L, Frielink C, Bouckenooghe T, Rijken P, Andralojc K, Göke BJ, de Jong M, Eizirik DL, Béhé M, Lahoutte T, Oyen WJG, Tack CJ, Janssen M, Boerman OC, Gotthardt M (2014) Non-invasive quantification of the beta cell mass by SPECT with ${ }^{111}$ In-labelled exendin. Diabetologia 57:950-959

42. Gotthardt M, Lalyko G, van Eerd-Vismale J, Keil B, Schurrat T, Hower M, Laverman P, Behr TM, Boerman OC, Göke B, Béhé M (2006) A new technique for in vivo imaging of specific GLP-1 binding sites: first results in small rodents. Regul Pept 137:162-167

43. Lacy PE, Hegre OD, Gerasimidi-Vazeou A, Gentile F, Dionne K (1991) Maintenance of normoglycemia in diabetic mice by subcutaneous xenografts of encapsulated islets. Science 254:1782-1784

44. Kerby A, Bohman S, Westberg H, Jones P, King A (2012) Immunoisolation of islets in high guluronic acid barium-alginate microcapsules does not improve graft outcome at the subcutaneous site. Artif Organs 36:564-570

45. Kawakami Y, Iwata H, Gu Y, Miyamoto M, Murakami Y, Yamasaki T, Cui W, Ikada Y, Imamura M, Inoue K (2000) Modified 
subcutaneous tissue with neovascularization is useful as the site for pancreatic islet transplantation. Cell Transplant 9:729-732

46. Juang JH, Bonner-Weir S, Ogawa Y, Vacanti JP, Weir GC (1996) Outcome of subcutaneous islet transplantation improved by polymer device. Transplantation 61:1557-1561

47. Pileggi A, Molano RD, Ricordi C, Zahr E, Collins J, Valdes R, Inverardi L (2006) Reversal of diabetes by pancreatic islet transplantation into a subcutaneous, neovascularized device. Transplantation $81: 1318-1324$

48. Golocheikine A, Tiriveedhi V, Angaswamy N, Benshoff N, Sabarinathan R, Mohanakumar T (2010) Cooperative signaling for angiogenesis and neovascularization by VEGF and HGF following islet transplantation. Transplantation 90:725-731

49. Barnett BP, Ruiz-Cabello J, Hota P, Liddell R, Walczak P, Howland V, Chacko VP, Kraitchman DL, Arepally A, Bulte JWM (2011) Fluorocapsules for improved function, immunoprotection, and visualization of cellular therapeutics with MR, US, and CT imaging. Radiology 258:182-191

50. Veriter S, Aouassar N, Adnet PY et al (2011) The impact of hyperglycemia and the presence of encapsulated islets on oxygenation within a bioartificial pancreas in the presence of mesenchymal stem cells in a diabetic Wistar rat model. Biomaterials 32:5945-5956 